\title{
PERCOLATION EFFECTS IN ELECTRICAL CONDUCTIVITY OF CARBON FIBRE COMPOSITES
}

\author{
R.D.Chippendale*, I.O.Golosnoy* \\ * School of Electronics and Computer Science, \\ University of Southampton, University Road, SO17 1BJ, UK \\ Contact email (RDC): rc805@ecs.soton.ac.uk
}

\section{Keywords: Conductivity, Carbon Fibre Composite, Percolation}

\begin{abstract}
The effects due to percolation on the bulk electrical conductivity of Carbon Fibre Composites are studied in detail. To simulate the CFCs manufacturing process the fibres are placed randomly in the polymer matrix using Monte Carlo based simulation techniques. The electric conductivity of the CFCs was then analysed using finite element modelling.
\end{abstract}

\section{Introduction}

Carbon Fibre Composites (CFCs) consist of two components with very different properties. These components are combined together to obtain a material suitable for a wide range of applications [4]. Normally CFCs contain several laminates arranged in plain layers. Each laminate has continuous carbon fibres aligned along one direction. The large difference in conductivity between the polymer and carbon fibre results in highly anisotropic characteristics in the individual laminates [7]. Electric conductivity of Carbon Fibre Composites (CFCs) depends strongly on fibre volume fraction. Since the polymer matrix has a low conductivity, the major conduction takes place through the carbon fibres, hence the percolation through the fibres [6] is important. It is known that percolation begins to affect the conductivity when the fibre volume fraction reaches a particular value, which depends on fibre shape, orientation and the production process $[3,5]$. The percolation threshold is considered in this paper by numerical studies of current flows, using a finite element model.

The majority of previous studies have either investigated the conductivity of polymeric materials with different fractions of highly conductive fillers or the percolation effects of short fibre composites $[6,8]$. In this paper we investigate the conductivity of long fibre composite materials whilst addressing the percolation phenomena.

\section{CFCs Materials Properties and Percolation Effect}

The carbon fibres in CFCs have a typical diameter between 8-10 $\mu \mathrm{m}$ [1] and a fibre volume fraction of $50-60 \%$. As a first approximation, it is possible to assume that the conductivities of the constituent parts of CFCs are constant and isotropic. The conductivity of carbon fibre and polymer is given by $\sigma_{f}$ and $\sigma_{p}$, respectively. It has been observed that $\sigma_{f}$ is $10^{10}-10^{20}$ larger than $\sigma_{\mathrm{p}}$ [8]. Initially, when the more conductive fibres are incorporated into the polymer the bulk conductivity for composite $\sigma_{\text {cfc }}$ will begin to gradually increase from the matrix value $\sigma_{p}$. At a given volume fraction of fibres, called the percolation threshold $\left(\varphi_{\text {perc }}\right)$, the bulk conductivity is expected to rapidly increase, as shown in previous studies $[6,8]$. Often, a power law is used to describe the phenomenon with the threshold and the power, to be fitted parameters, as discussed in [8]. The objective of this paper is to investigate these parameters for a typical CFC. This is done numerically to avoid expensive experimental studies.

\subsection{Modelling Method}

By considering a thick single layer laminate with perfectly straight cylindrical carbon fibres of constant radius, all orientated in the same direction, the conduction problem can be modelled as a $2 \mathrm{D}$ cross section slice, due to the symmetry entailed. To simulate the CFCs manufacturing process, the fibres are placed randomly in the polymer using Monte Carlo based simulation techniques. The polymer is depicted by a rectangular box with unit dimension in $x-$ and $y$ directions. Two different modelling approaches where considered.

\subsection{Monte Carlo Fibre Placement Model}

Using the 2D geometry, fibres are considered to be perfect circles. Each circle in positioned by generating two random numbers which are used to describe the centre. A fixed number of circles are positioned, so the volume fraction of fibres is controlled by fibres radius.

Each new circle is checked against all previous circles to determine if its location is valid. The validity is determined by checking that the entire circle lies inside the bounds of the polymer, and that if two circles do overlap it is limited to $10 \%$ of the circles radius. This process is repeated until there are a predefined number of circles (i.e. fibres) in the model. A geometrical representation of our model is shown in Figure 1.

\subsection{Monte Carlo Fibre Removal Model}

With the previous method it is difficult to obtain a high fibre volume fraction (above $\sim 60 \%$ ). To obtain higher volume fractions the simulation starts with the perfect fibre packing, which produces the highest possible volume fraction. Then a specified number of random 
circles (fibres) are removed leaving polymer in their place.

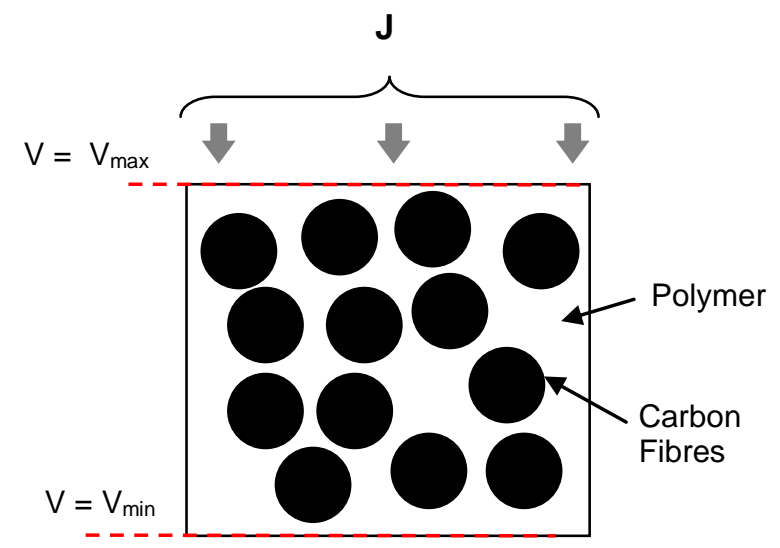

Figure 1. 2D representation of the model geometry

\subsection{Governing equations and boundary conditions}

As shown in Figure 1. The numerical model is solved with a potential difference applied across the sample. A steady state current flow problem is well described by standard Ohm's law. In terms of the electric current density $\mathbf{J}$ and the electric field $\mathbf{E}$ it is given by Equation (1), which in turn results in formulation for the electric potential $V$ :

$$
\nabla \cdot \mathbf{J}=0, \mathbf{J}=\sigma_{c f_{c}} \mathbf{E}, \mathbf{E}=-\nabla V
$$

Assuming the potential difference decrease through the material is linear the bulk conductivity of the composite can be calculated using Equation (1). The averaged current density, required by the formula, can be determined from the numerical solution.

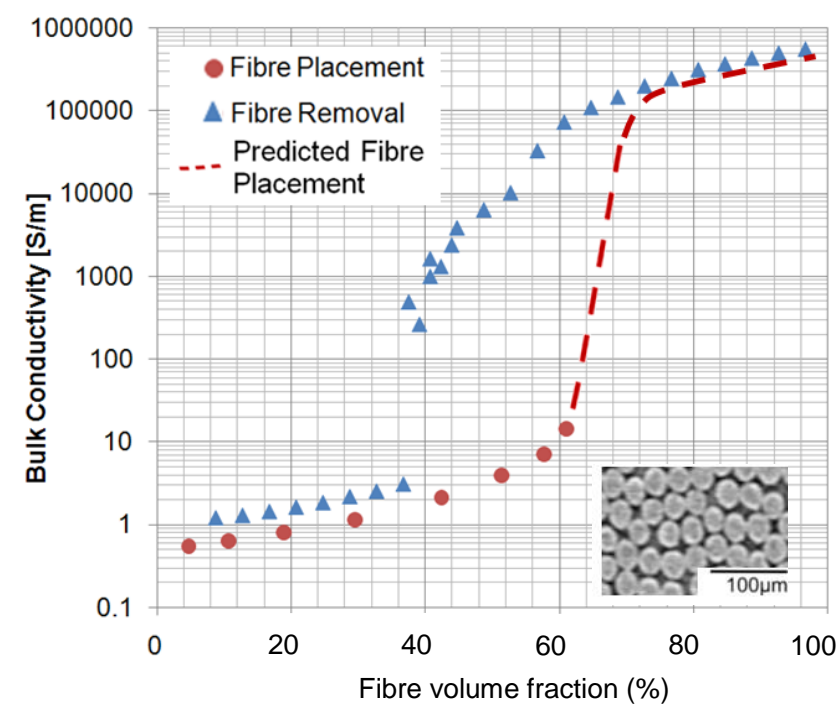

Figure 2. Results of bulk electrical conductivity $\left(\sigma_{\mathrm{cfc}}\right)$ from the two numerical modelling model. Also shown is a cross section of typical CFC

For every given volume fraction within each model, the simulations are repeated to collate an average bulk conductivity $\left(\sigma_{\mathrm{cfc}}\right)$ and corresponding error.
Numerical predictions were obtained from a commercial finite element software COMSOL [2]. Triangular mesh elements and a linear discretization was used.

\section{Results}

The conductivities where taken to be $\sigma_{\mathrm{f}}=1 \times 10^{6} \mathrm{Sm}^{-1}$ and $\sigma_{p}=1 \mathrm{Sm}^{-1}$. The numerical predictions from the two modelling methods are shown in Figure 2.

Both models show that at low volume fractions there is minimal change in bulk conductivity. As the fibre volume fraction increases the bulk conductivity begins to increase. The predicated percolation threshold given by the fibre removal model is $\varphi_{\text {perc }}=40 \%$, whereas from the fibre placement model $\varphi_{\text {perc }}=60 \%$. The fibre removal model is considered the most realistic when considering a true fibre arrangement, shown in Figure 2. In the extended version of the paper specific attention will be focused on investigating the variation of polymeric conductivity on the bulk conductivity along with attempting to fit a power law as in previous studies.

\section{Acknowledgements}

This work has been partially supported by EADS UK, through an industrial studentship. Thanks to Prof. Ian Sinclair at the University of Southampton for the CFC cross section tomography image.

\section{References}

1. D. Chung. "Carbon Fiber Composites". (1994): Butterworth-Heinemannp.

2. COMSOL Inc, COMSOL Multiphysics simulation environment: Stockholm, Sweden.

3. G. E. Pike and C. H. Seager. "Percolation and conductivity: A computer Study I*", Phys. Rev. B, 10(4), pp. 1421-1434, (1974).

4. S. M. Lee. "International Encyclopedia of Composites". (1990): New York.

5. M. M. Tomadakis and S. V. Sotirchos. "Transport properties of random arrays of freely overlapping cyclinders with various orientation distributions", J. Chem. Phys., 98, pp. 616-626, (1993).

6. M. Taya and N. Ueda. "Prediction of the In-Plane Electrical Conductivity of a Misoriented Short Fiber Composite: Fiber Percolation Model Versus Effective Medium Theory", J. Eng. Mater. Technol. , 109(3), pp. 252-256 (1987).

7. R. Rolfes and U. Hammerschmidt. "Transverse thermal conductivity of CFRP laminates: A numerical and experimental validation of approximation formulae", Comp. Sci. Techn., 57, pp. 227-285, (1997).

8. Ye. P. Mamunya et. al. "Electrical and thermal conductivity of polymer filled with metal powders", European Polymer Journal, 38, pp. 1887-1897, (2002). 